http://jmscr.igmpublication.org/home/ ISSN (e)-2347-176x ISSN (p) 2455-0450

crossref DOI: https://dx.doi.org/10.18535/jmscr/v8i3.109

Journal Of Medical Science And Clinical Research

IGM Publication

An Official Publication of IGM Publication

\title{
Class II Correction with Forsus Fixed Functional Appliance in Class II Division 2 Malocclusion: A Case Report
}

\author{
Authors \\ Dr Irum Uzma Siddiquie ${ }^{1}$, Dr Saba Khan², Dr Muhammed Shareef Parakkal ${ }^{3}$, \\ Dr Mohd Zubair Ansari ${ }^{4}$ \\ ${ }^{1,2,3,4}$ Department of Orthodontics and Dentofacial Orthopaedics, Dr Ziauddin Ahmad Dental College/ \\ Aligarh Muslim University, India
}

\begin{abstract}
Case Report of a 14 year old male patient reported with class II division 2 malocclusion with increased overbite, decreased over jet, retroclined upper anteriors, concordant midline, convex facial profile, posterior divergence on skeletal class II base with hypo divergent growth pattern and CVMI stage IV. this case was treated with utility arch and MBT prescription for the duration of 2 year, class II correction is achieved with the help of Forsus fixed functional appliance. The result showed correction of overjet and overbite, with class I molar and canine relation and marked improvement in facial esthetics

Keywords: Class II Div 2, class II correction, Forsus fixed functional appliance.
\end{abstract}

\section{Introduction}

Class II malocclusion presents a major and common challenge to orthodontic practice that exhibits maxillary protrusion, mandibular retrognathism or combination of both, with abnormal dental relationship and facial aesthetic disorders. Treatment modalities are chosen based on the consideration of skeletal, dental and soft tissue factors. Functional orthopedic appliances are mostly used to treat Class II malocclusion originated from mandibular retrusion. Non complaint patients and patient who already passed their growth spurt are mostly treated with fixed functional appliances. One of the most preferred compliance free fixed functional appliances is Forsus Fatigue Resistant Device (FRD, 3M Unitek, Monrovia, Calif) which is a three piece telescopic system incorporated with super elastic nickel- titanium coil spring coming under the catogery of Hybrid appliance ${ }^{1}$

\section{Case Report}

A 13 year old male reported to the department of orthodontics with complaint of irregularly placed upper and lower front teeth with familial history of class II div 2 malocclusion. He had no history of habit and relavant medical history.

On extraoral examination, the patient had apparently symmetrical mesoprosopic face with convex profile, posterior divergence \& competent lips. Smile analysis showed a high smile line with a non-consonant smile arc and morley's ratio of $100 \%$ (Figure 1). Upon intraoral examination the patient had class II molar and canine relationship bilaterally, an overjet of $1 \mathrm{~mm}$, overbite of $80 \%$, occlusal features showed symmetric U-shaped maxillary arch and an ovoid shaped mandibular arch with mild crowding in lower anterior region. (Figure 1) The periodontal tissues were found to be healthy. The functional examination showed 
no signs or symptoms of a temporomandibular joint disorder.

Cephalometric findings presented as a forwardly placed maxilla with retrognathic mandible resulting in skeletal class II relation $\left(\mathrm{ANB}=5^{\circ}\right.$, Wits $=4.5 \mathrm{~mm}$, APP-BPP $=8 \mathrm{~mm}, \mathrm{MM}$ bisector $=$ $3 \mathrm{~mm})$. The patient had a hypodivergent growth pattern as showed by FMA $20^{\circ}$, SN-MP $22^{\circ}$, Y
Axis $55^{\circ}$, Bjork's sum $383^{\circ}$. Gonial angle $113^{\circ}$ along with retroclined upper and normally placed lower incisors (Max 1-APog $=5 \mathrm{~mm}$, Max 1$\mathrm{NA}=17^{\circ} \& 3 \mathrm{~mm}$, Mand 1-APog $=0 \mathrm{~mm}$, Md I $-\mathrm{NB}$ $\left.=4 \mathrm{~mm} \& 23^{0} \& \mathrm{IMPA}=100^{\circ}\right)$. Soft tissue cephalometric analysis revealed little protrusive upper and lower lip w.r.t S and E line, nasolabial angle was $100^{\circ}$ without lip strain (Figure 2).

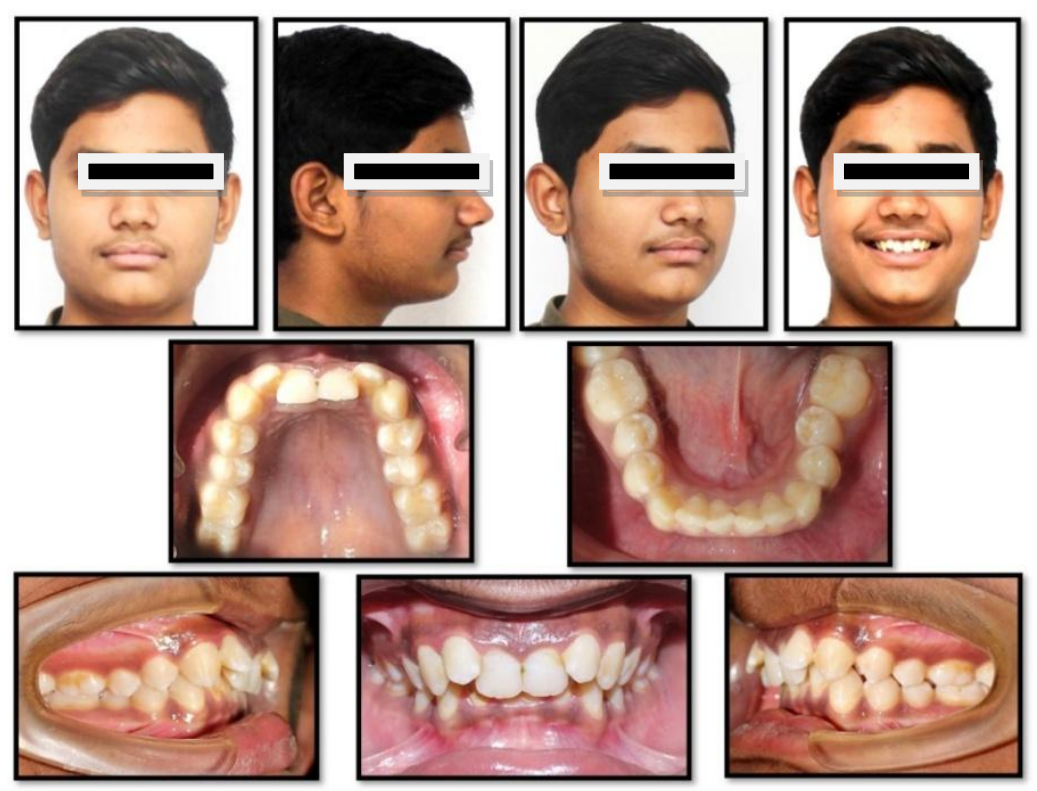

Figure 1: Pre-treatment photographs
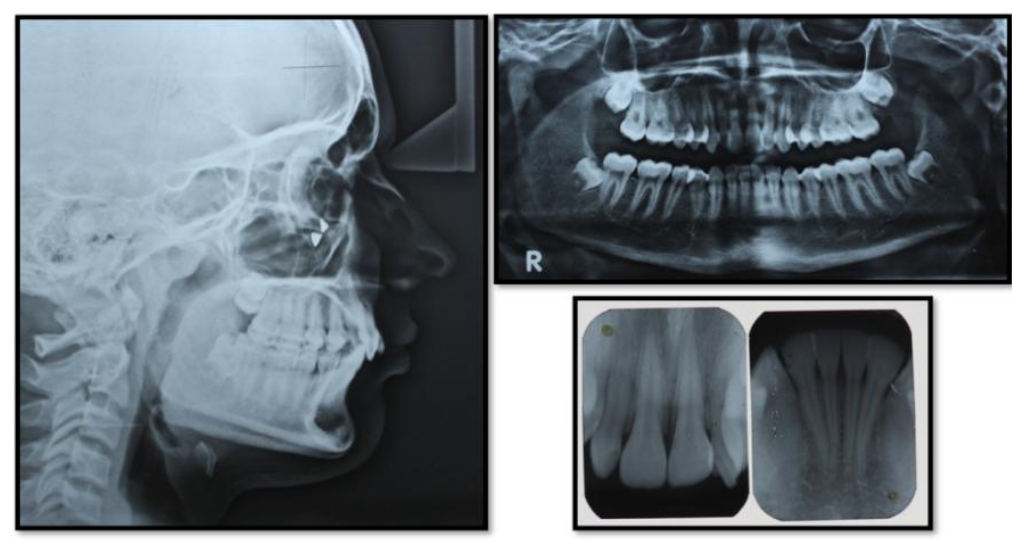

Figure 2: Pre-treatment radiographs

The panoramic X-ray showed the presence of upper and lower third molars in all quadrant with no apparent pathologies (Figure 2). Study Model analysis showed a total discrepancy of $2 \mathrm{~mm}$ in the upper arch and $-1 \mathrm{~mm}$ in the mandibular arch.

\section{Diagnosis and Treatment Objectives}

An 13 year old male with chief complaint of irregularly placed upper and lower front teeth has Angle's class II molar and canine relation bilaterally with class II Division 2 incisor pattern, decreased overjet, increased overbite on skeletal class II base with hypodivergent growth pattern and CVMI-IV.

The goal was to obtain a good facial balance with optimum static and functional occlusion. The treatmentobjectives were:

- Correction of class II skeletal base

- Correction of retroclined upper incisors 
- Correction of deep bite

- Achieving class I molar and canine relation bilaterally.

- correction of overjet and overbite

- Achieving stable occlusal function

- Achieving optimum soft tissue balance

- Long term retention

\section{Treatment Plan}

By considering cephalometric hard and soft findings as well as the study model analysis a conservative treatment plan was outlined with non extraction fixed orthodontic mechanotherapy

\section{Treatment Progress}

Treatment is started with a 2 X 2 utility arch in maxilla for intrusion and proclination for upper central incisors (Figure 3). 3 months later after proclining incisors both maxillary and mandibular arch were banded and bonded using preadjusted edgewise appliance (MBT 0.022x0.028 slot) and an initial 0.016" NiTi wire was placed for levelling and alignment of the arches. By 5 months, good levelling and alignment was achieved to place $0.019 \times 0.025$-inch SS wires in both upper and lower arch (Figure 4). $6 \mathrm{~mm}$ of overjet and $50 \%$ of overbite were remaining after levelling and alignment with class II molar and canine relation. Then a $32 \mathrm{~mm}$ Forsus Fixed functional appliance was delivered which fixed in molar tube in maxillary arch and distal to canine on mandibular arch wire for 5 months. With the treatment Marked reduction in overjet and overbite were noticed with significant improvement in the facial profile. Forsus was removed after achieving class I molar and canine relation on both the sides and an acrylic plate with reverse inclined plane was given to avoid relapse. Occlusal settling was done with 0.14 inch stainless steel wire with settling elastics. The total treatment took 18 months to settle a class II div 2 case in class I molar and canine relation with improved facial aesthetics.

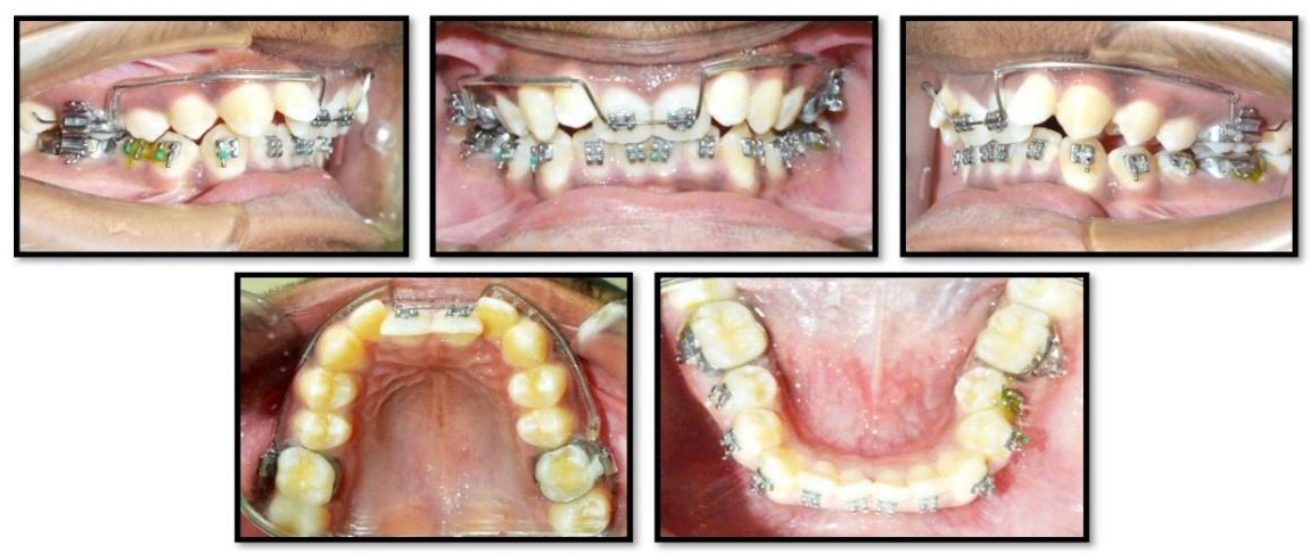

Figure 3: Utility arch for intrusion and proclination of retroclined incisors

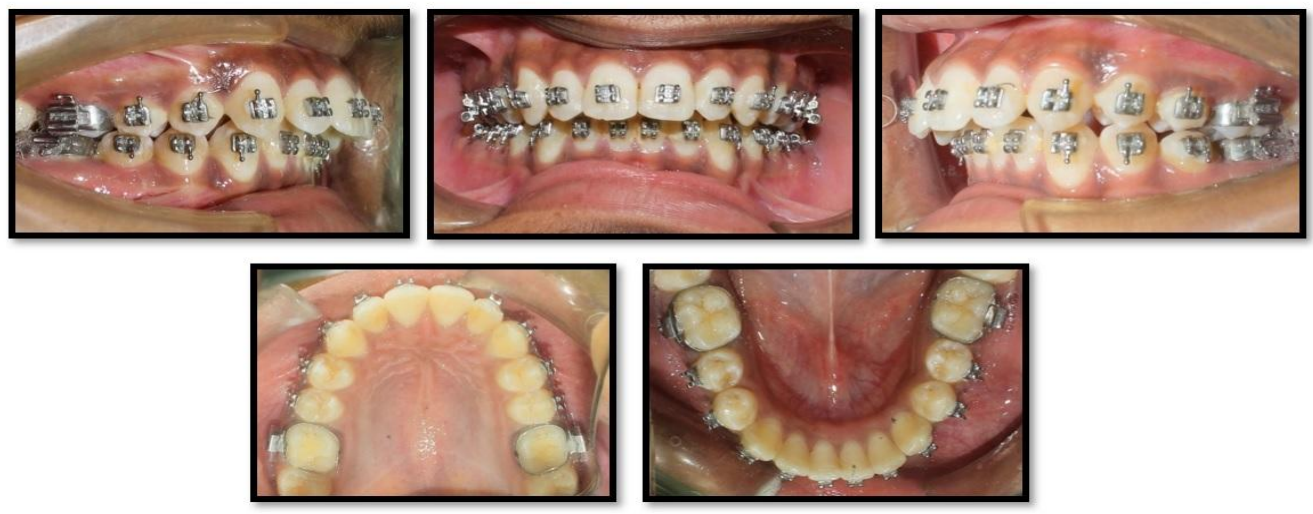

Figure 4: Levelling and alignment 

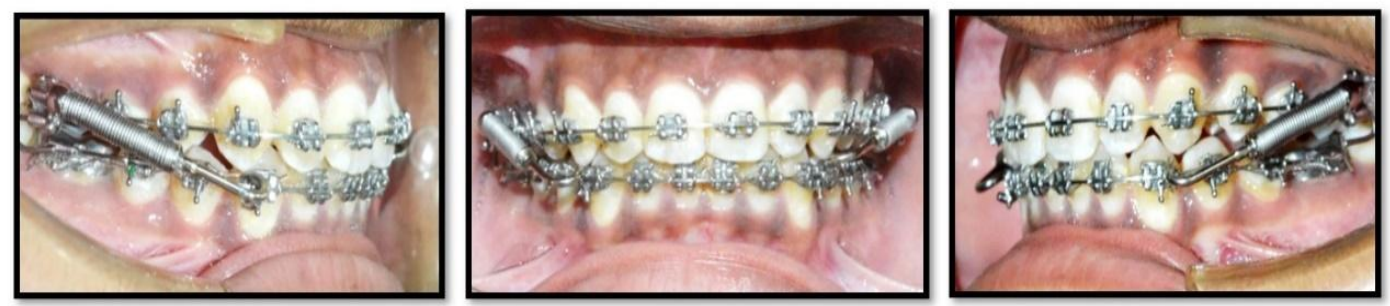

Figure 5: Forsus FRD Fixed Functional Appliance

\section{Treatment Results}

The facial aesthetic was improved with better lip support (Figure 6). The smile was enhanced and the consonant smile arc was achieved. Intraorally, ideal overjet and overbite was achieved with Class I molar and canine relationship (Figure 7). The post treatment panoramic radiograph showed good overall root parallelism and lack of root resorption.Post treatment lateral Cephalogram (Figure 8) showed no change in the skeletal
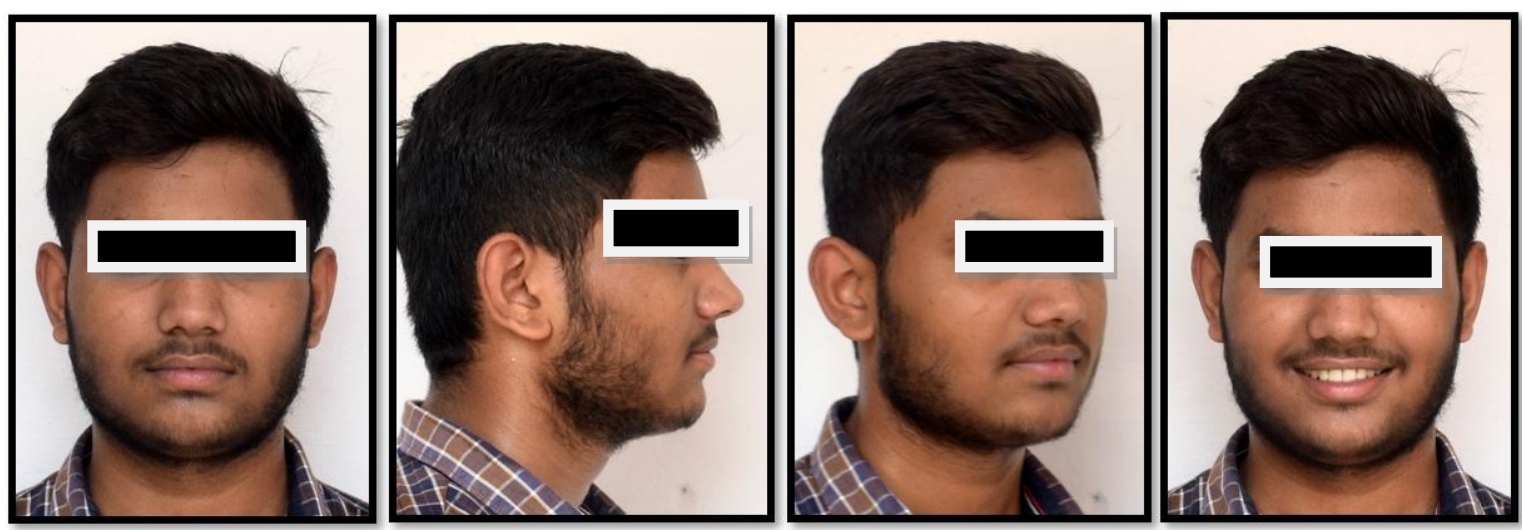

Figure 6: Post-treatment extraoral photographs
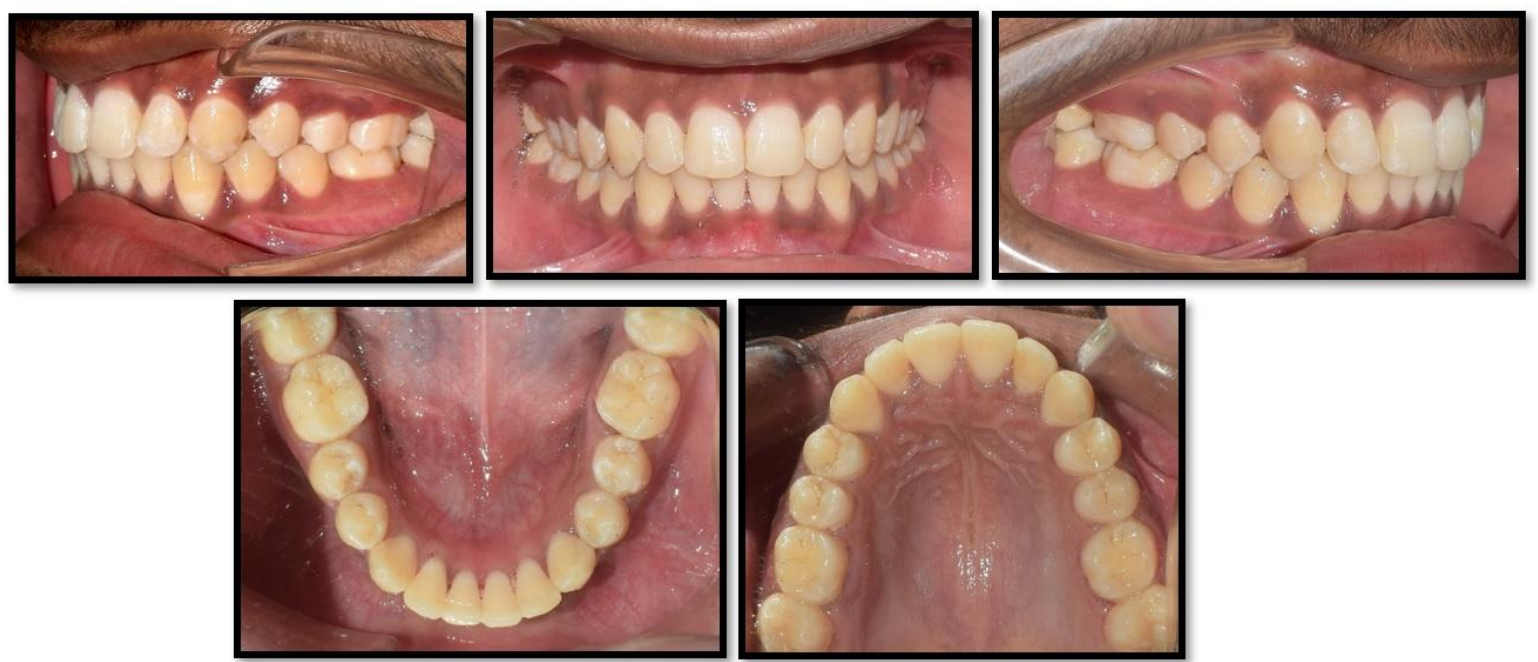

Figure 7: Post treatment Intraoral photographs 


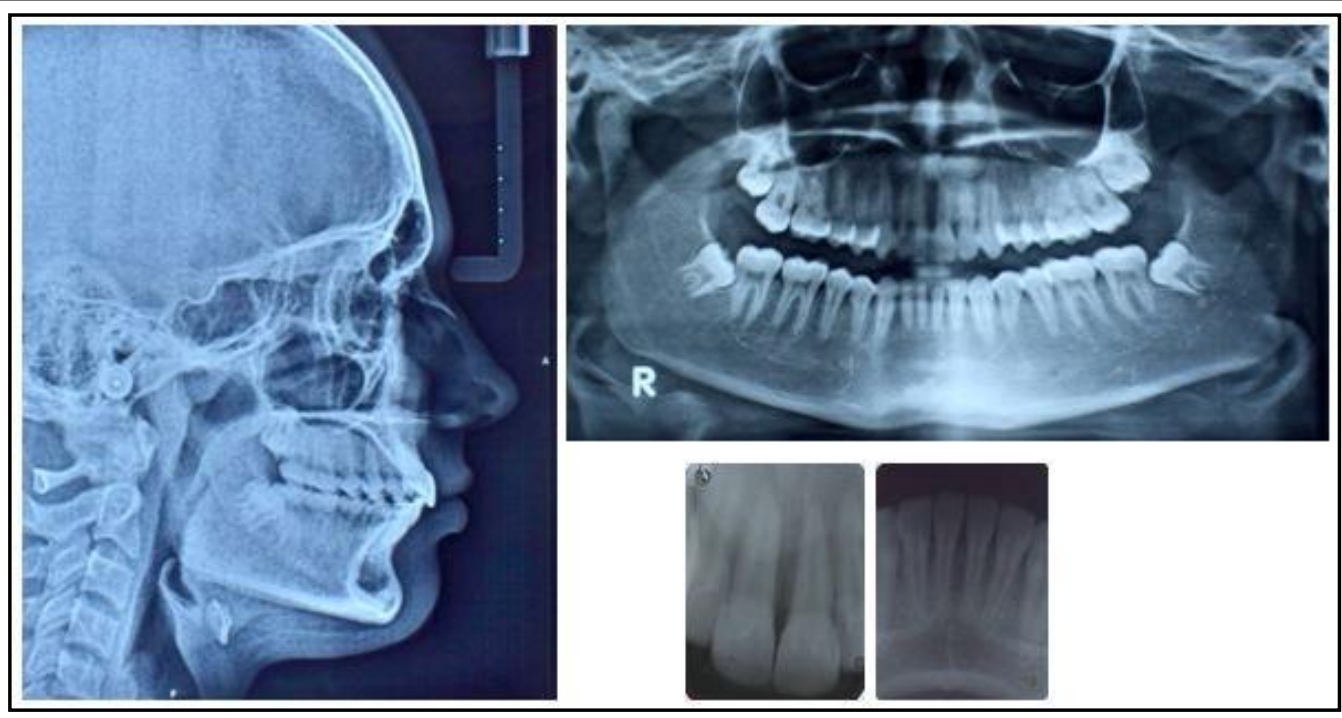

Figure 8: Post treatment radiographs

\begin{tabular}{|c|c|c|}
\hline Cephalometric parameters & Pre treatment & Post treatment \\
\hline SNA & $82^{0}$ & $81^{0}$ \\
\hline SNB & $77^{0}$ & $78^{0}$ \\
\hline ANB & $5^{0}$ & $3^{0}$ \\
\hline WITS & $4.5 \mathrm{~mm}$ & $3 \mathrm{~mm}$ \\
\hline $\mathbf{N} \mid \mathbf{A}$ point & $0 \mathrm{~mm}$ & $-2 \mathrm{~mm}$ \\
\hline N] B point & $-15 \mathrm{~mm}$ & $-12 \mathrm{~mm}$ \\
\hline \begin{tabular}{|l|l|}
$\mathbf{N}$ & Pogonion \\
\end{tabular} & $-13 \mathrm{~mm}$ & $-10 \mathrm{~mm}$ \\
\hline FMA & $20^{0}$ & $23^{0}$ \\
\hline SN-MP & $22^{0}$ & $28^{0}$ \\
\hline Bjork & $\mathbf{3 8 3}^{0}$ & $390^{0}$ \\
\hline Mx I to NA & $3 \mathrm{~mm}$ & $5 \mathrm{~mm}$ \\
\hline Mx I to NA & $17^{0}$ & $27^{0}$ \\
\hline Md I to NB & $0 \mathrm{~mm}$ & $4 \mathrm{~mm}$ \\
\hline Md I to NB & $23^{0}$ & $32^{0}$ \\
\hline IMPA & $100^{0}$ & $110^{0}$ \\
\hline E - line upper lip & $-2 \mathrm{~mm}$ & $-3 \mathrm{~mm}$ \\
\hline E - line lower lip & $0 \mathrm{~mm}$ & $2 \mathrm{~mm}$ \\
\hline Nasolabial angle & $100^{0}$ & $96^{0}$ \\
\hline
\end{tabular}

SO (Sagital occlusal) Analysis (Pancherz)

\begin{tabular}{|c|c|c|}
\hline $\begin{array}{l}\text { Variables } \\
\text { Linear Measurements }\end{array}$ & Pre treatment & Post treatment \\
\hline Maxillary Base (ss/RLp) & $79 \mathrm{~mm}$ & 78.5mm \\
\hline Mandibular Base (pg/RLp) & $74 \mathrm{~mm}$ & $76 \mathrm{~mm}$ \\
\hline Maxillary incisor (is/RLp) & $84 \mathrm{~mm}$ & $83 \mathrm{~mm}$ \\
\hline Mandibular incisor (ii/RLp) & $78 \mathrm{~mm}$ & $82 \mathrm{~mm}$ \\
\hline Maxillary Molar (ms/RLp) & $56 \mathrm{~mm}$ & $54.5 \mathrm{~mm}$ \\
\hline Mandibular molar (mi/RLp) & $53 \mathrm{~mm}$ & $57 \mathrm{~mm}$ \\
\hline Overjet & $6 \mathrm{~mm}$ & $1 \mathrm{~mm}$ \\
\hline Molar relation & $+3 \mathrm{~mm}$ & $-2.5 \mathrm{~mm}$ \\
\hline
\end{tabular}

\section{Retention}

Bonded lingual retainer for lower arch and removable Hawleys retainer with reverse inclined plane for the upper arch were given for retention.

\section{Discussion}

A functional appliance is an appliance that is designed to alter the neuromuscular environment of the orofacial region to improve occlusal development and craniofacial skeletal growth. 
Fixed functional appliances are reduces the need for patient compliance and brings the outcome of the treatment in the hands of orthodontist. Though the class II correction achieved in this patient was due to changes in both skeletal and dentoalveolar parameters, changes in dentoalveolar structures were pronounced which include mesial movement of the mandibular molars, distal movement and intrusion of maxillary molar and proclination and intrusion of lower incisors etc. Changes were noticed both in anteroposterior and vertical plane because of the attachment of Forsus that provides a force vector on maxillary dentition in a backward and upward direction and on lower anteriors in a forward and downward direction. Proclination of the lower incisors and retroclination of upper incisor were the significant contributing factor for overjet reduction. The similar result was noticed by Esen Ali Gunay $(2011)^{2} \&$ Aslan $(2014)^{3}$ in their study with Forsus in which class II correction was achieved by dentoalveolar changes without any skeletal changes. Though the patient was in post pubertal growth spurt, he had shown mild skeletal changes both in the anteroposterior and vertical plane which was due to headgear effect of Forsus appliance with restraining effect on maxilla and sagittal advancement in mandibule. Cacciator $(2014)^{4}$ had seen improvement in the SNB angle and mandibular growth during circumpubertal age by the use of Forsus FRD. Vertical skeletal changes seen during the treatment were opening of gonial angle, increaded FMA and increase in anterior facial height. Graham Jones (2008) ${ }^{\mathbf{5}}$ studied the effect of class II elastic and Forsus FRD and concluded that Class II elastics are also effective in correcting Class II malocclusions, and their effects are primarily dentoalveolar. They are similar to the effects of fixed functional appliances in the long term, Forsus is more effective for correcting Class II malocclusion in a shorter treatment period with minimal patient compliance required.

\section{Conclusion}

The hybrid fixed functional appliance such as Forsus FRD is a successful treatment protocol for correction of class II malocclusion. The changes brought by the appliance was mainly dentoalveolar with minimal skeletal effect and providing a shorter treatment period and requiring minimal patient compliance. More skeletal changes can be expected in those patients treated in their active growth period like in peak height velocity.

\section{References}

1. Franchi, L., Alvetro, L., Giuntini.V., Masucci, C., Defraia,E., Baccetti,T. Effectiveness of comprehensive fixed appliance treatment used with the Forsus Fatigue Resistant Device in Class II patients. Angle Orthodontist,2011, Vol 81, No 4

2. Gunay, EA., Arun,T., Nalbantgil, D. Evaluation of the Immediate Dentofacial Changes in Late Adolescent Patients Treated with the Forsus FRD. Eur J Orthod 2011;5;423-432

3. Aslan,B., Kucukkaraca, E., Turkoz, C., Dincer, M. Treatment effects of the Forsus Fatigue Resistant Device used with miniscrew anchorage. Angle Orthod 2014; 84(1;,76-86

4. Cacciatore G, Ghislanzoni HT, Alvetro L, Franchi L. Treatment and post treatment effects induced by the Forsus appliance a controlled clinical study. Angle Orthod 2014; 84:1010-17

5. Jones $\mathrm{G}$ et al, Class II Non-Extraction Patients Treated with the Forsus Fatigue Resistant Device Versus Intermaxillary Elastics. Angle Orthodontist, Vol 78, No 2, 2008

6. Mahamad IK, Neela PK, Husain MR. A comparison of Twin Block and Forsus appliance a cephalometric study. Int $\mathbf{J}$ Orthod Milwaukee 2012; 23(3):49-58. 
7. Oztoprak MO, Nalbantgil D, Uyanar A, Arun T. A cephalometric comparative study of class II correction with Sabbagh Universal Spring and forsus FRD appliance. Eur J Dent 2012;6 :302-10

8. Bowman AC, Saltaji H, Mir FC, Preston B, Tabbaa S. Patients experience with the Forsus Fatigue Resistant Device. Angle Orthod 2013;83:437-46.

9. Perineti G, Primozic J, Furlani G, Franchi L, Contrado L. Treatment effects of Fixed Functional Appliance alone or in combination with multi bracket appliance. Angle Orthod 2015; 85:480-92.

10. Servello DF, Fallis DW, Alvetro L. Analysis of Class II patients, successfully treated with the straight-wire and Forsus appliances, based on cervical vertebral maturation status. Angle Orthod 2015;85:80-86. 